
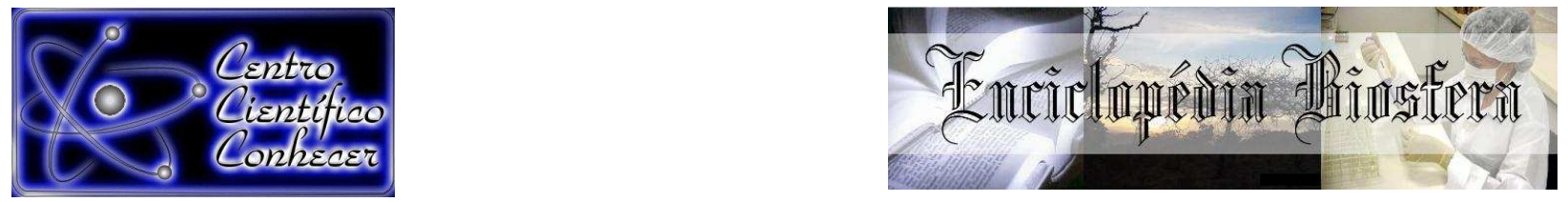

\title{
ANÁLISE DO COMPORTAMENTO DOS PREÇOS DAS PRINCIPAIS FRUTAS E HORTALIÇAS-FRUTO COMERCIALIZADOS NA CEASA-CE
}

\footnotetext{
Alfredo Mendonça de Sousa1, Francisco José Silva Tabosa²; Antônio Moreira

Barroso Neto3; Francisco Casimiro Filho2; José Newton Pires Reis²

1Pós-Graduando do Programa de Mestrado em ciências do solo, na Universidade

Federal do Ceará, Campus do Pici, Fortaleza, Ceará (E-mail:

alfredomendonca121@gmail.com)

2Professor Doutor do Curso de Agronomia (Departamento de Economia Agrícola) na

Universidade Federal do Ceará, Campus do Pici, Fortaleza, Ceará

3Pós-Graduando do Programa de Mestrado em Fitotecnia, na Universidade Federal do Ceará, Campus do Pici, Fortaleza, Ceará

Recebido em: 08/04/2016 - Aprovado em: 30/05/2016 - Publicado em: 20/06/2016 DOI: 10.18677/Enciclopedia_Biosfera_2016_007
}

\begin{abstract}
RESUMO
A agricultura, especialmente o setor de fruticultura, possui considerável importância na economia cearense e do Nordeste como um todo, apontando para a necessidade do conhecimento de características relacionadas à comercialização de frutas assim como estudos sobre o comportamento dos preços destes produtos. O conhecimento do padrão de sazonalidade e tendência dos preços dos produtos originários deste setor possui significativa importância no contexto de planejamento agrícola. Assim, este trabalho teve como objetivo analisar o comportamento dos preços das principais frutas e hortaliças-fruto comercializados na CEASA-CE. Para isso, filtrou-se as culturas ofertadas na CEASA-CE, Sede Maracanaú, originárias no Estado do Ceará, obtendo-se, desta forma, os principais produtos produzidos no Estado que são comercializados na Sede citada. Obteve-se o histórico de preços para o período de 2002 a 2014 de cada cultura analisada, procedendo-se a deflação destes valores com base no IGP-DI calculado pela FGV, base de dezembro de 2014. Utilizou-se o método da média móvel centralizada para identificar os índices sazonais das culturas. Procedeu-se, em seguida, a análise de tendência mensal dos históricos de preços analisados. Todas as culturas demonstraram padrão sazonal de variação de preços e as culturas de tomate, melancia, pimentão, melão japonês e limão apresentaram tendência de queda dos preços.
\end{abstract}

PALAVRAS-CHAVE: Planejamento Agrícola. Sazonalidade. Tendência de Preços.

\section{ANALYSIS OF BEHAVIOR OF PRICE OF FRUIT AND VEGETABLES MAIN-FRUIT MARKETED IN CEASA-CE}

\begin{abstract}
The agriculture, especially the fruit growing sector, possesses considerable importance in Ceará economy and to the Nordeste as a whole, pointing to the need to know the characteristics related to the marketing of fruit as well as studies on the behavior of prices of these products. The knowledge of seasonality pattern and trend ENCICLOPÉDIA BIOSFERA, Centro Científico Conhecer - Goiânia, v.13 n.23; p. 72 2016
\end{abstract}


the prices the products originating from this sector, has significant importance in the context of agricultural planning. So, this work had as objective analyze the behavior the prices of the main fruits and vegetables-fruits marketed in the CEASA-CE. For this, filtered itself the cultures tendered in the CEASA-CE, Headquarters Maracanaú, originating in the State of Ceará, getting itself, so, the main products produced in the State that are marketed in the Headquarters quoted. There was obtained price history for the period of 2002 to 2014 of each culture analyzed, proceeding itself the deflation of these values based on the IGP-DI calculated by FGV, base of december of 2014. Utilized itself the method of centralized moving average to identify the seasonal indices of cultures. Proceeded itself, then, the analysis monthly trend of price history analized. All cultures demonstrated seasonal pattern of price variation and the cultures of tomato, watermelon, pepper, Japanese melon and lemon presented downward trend of price.

KEYWORDS: Seasonality. Price Trend. Agricultural Planning.

\section{INTRODUÇÃO}

A economia do Ceará cresceu significativamente nos últimos anos, e dentre os setores que mais se desenvolveram destaca-se a agricultura, especialmente a fruticultura, que é atualmente um dos principais setores em crescimento na região, gerando emprego e renda para a população (FACHINELLO et al., 2011). Inseridos dentro deste contexto de aumento de produção de alto risco econômico estão os produtores, as indústrias, os atacadistas e consumidores finais em geral na condição de demandantes finais destes produtos (HARZER et al. 2012).

O Estado do Ceará possui hoje uma produção agrícola diversificada. Dentre as culturas produzidas destacam-se, em volume de comercialização, por exemplo, banana pacovan, tomate, banana prata, mamão formosa e melancia. Essas culturas, em 2013, atingiram os maiores volumes de comercialização na CEASA (Central de Abastecimento do Ceará), Sede Maracanaú, referentes a produtos oriundos do Estado do Ceará (CEASA, 2013).

As flutuações ou variações estacionais dos preços de um produto agrícola são geralmente ligadas a um padrão comum de comportamento nos mesmos meses em anos sucessivos (MARQUES \& AGUIAR, 1993). Entretanto, além da variação estacional de uma série de preços, podem existir, em determinados anos, movimentos irregulares ou aleatórios relativos a eventos casuais (SPIEGEL, 1993).

Levando-se em consideração as expressivas variações nos preços de produtos agrícolas, o conhecimento da variação estacional e da tendência, tornamse importantes ferramentas de tomada de decisão para produtores, comerciantes, consumidores, varejistas e também para o Governo.

É razoável, portanto, considerar que o conhecimento das variações sazonais dos preços agrícolas, assim como o conhecimento das tendências de preços, possui relevante importância principalmente tendo em vista os movimentos de oferta e demanda. O conhecimento das variações sazonais dos preços, por exemplo, fornece subsídios aos produtores para alocação temporal de recursos mais eficiente. Além disso, pode servir para orientar o consumidor sobre as melhores épocas de compra, melhorando a eficiência da utilização da renda (MARQUES \& CAIXETA FILHO, 2002).

Vários estudos foram realizados com foco no comportamento de preços agrícolas, devido à necessidade de atualização constante das informações contidas nestas publicações, tornando esta área atraente para o campo de estudo e 
pesquisa. Estudos nesta linha são observados nos trabalhos de BENTO \& TELES (2013) e CARDOSO et al. (2013), por exemplo.

MARQUES \& AGUIAR (1993) citam que a melhor época do ano para se adquirir o produto, organizar os estoques a fim de amenizar o impacto das elevações nos preços na época de queda na oferta, formulação de políticas de renda mínima, aquisição de crédito bancário, entre outros, são algumas das decisões que poderiam ser diretamente afetadas com o conhecimento da variação estacional de preços de um dado produto agrícola de interesse. Por tais motivos, objetivou-se analisar no presente trabalho o comportamento dos preços das principais frutas e hortaliçasfruto comercializados na CEASA-CE.

\section{Fonte de Dados}

\section{MATERIAL E MÉTODOS}

A base de dados utilizada para a construção dos índices e indicadores necessários à realização das análises de sazonalidade e tendência dos preços dos principais produtos agrícolas foi o histórico de oferta e preços de comercialização destes produtos, disponíveis no Site oficial das Centrais de Abastecimento do Ceará (CEASA).

Para realizar a seleção das culturas a serem analisadas, filtraram-se os dados disponibilizados no Site da CEASA com base no estado de origem. Este procedimento permitiu selecionar as 10 principais culturas comercializadas no CEASA, com base no volume de comercialização, são elas: banana pacovan, tomate, banana prata, mamão formosa, melancia, pimentão, maracujá, chuchu, melão japonês e limão. Vale salientar que a décima cultura, em volume de comercialização, foi a manga coité, no entanto, para esta cultura, não estão disponíveis histórico de preços. Desta forma, substituiu-se a manga coité pela cultura do limão, que é a décima primeira cultura em volume de comercialização.

O estudo contempla o período de janeiro de 2002 a dezembro de 2014, estando os preços em bases mensais para todo o período analisado.

\section{Análise de Sazonalidade}

Para a realização da análise de sazonalidade utilizou-se, a fim de determinar os índices estacionais de preços, o método da média aritmética móvel, exemplos da aplicação deste método são encontrados em MENDES \& PADILHA JÚNIOR (2007), BARROSO NETO et al. (2015) e SOUSA et al. (2015).

As séries de preços calculados pelo sistema de informação da CEASA e disponibilizado em tabelas de histórico de preços foram deflacionadas através do Índice Geral de Preços - Disponibilidade Interna - (IGP-DI) calculados pela Fundação Getúlio Vargas - (FGV).

Após realizada a deflação do histórico de preços, determinou-se as médias aritméticas móveis centrada em doze meses. A média móvel centralizada foi calculada para todos os meses a partir do mês de julho de 2002 até o mês de junho de 2014.

Com as médias determinadas calculou-se o IEP (índice estacional de preço) através da seguinte fórmula: 
$I E P=\left(\frac{P}{M M C}\right) * 100$

Em que,

IEP = Índice estacional de Preço, em \%;

$\mathrm{P}=$ Preço no mês analisado, em $\mathrm{R} \$$;

MMC = Média Móvel Centralizada, em R\$.

Após o cálculo do IEP, procedeu-se a determinação do índice sazonal. Para isso, calculou-se inicialmente o IEP médio para cada cultura considerando os 12 meses do ano, nos casos em que o valor da média calculada não foi igual a 100, cada índice mensal foi ajustado por meio da multiplicação de um fator de correção conveniente, definido através da seguinte equação:

Fator de Correção $=\left(\frac{100}{\text { IEPmé dio }}\right)$

Após o cálculo do fator de correção, procedeu-se a multiplicação deste pelo IEP de cada mês, obtendo-se, assim, os índices de sazonalidade. Em seguida, foi determinado os limites superiores e inferiores de cada mês para todo o período analisado.

\section{Análise de Tendência}

Visando obter a tendência anual de crescimento dos preços das culturas analisadas, utilizou-se o método linear. $O$ modelo linear consiste em ajustar uma equação de primeiro grau em uma série de preços para verificação do comportamento dos mesmos ao longo do tempo, conforme equação à seguir:

$$
P t=a \pm b . t
$$

Em que,

$\mathrm{Pt}=$ preço do produto agrícola no tempo t (anos);

$\mathrm{a}=$ intercepto ou coeficiente linear;

b = inclinação ou coeficiente angular;

$\mathrm{t}=$ tendência.

O componente $t$ (tendência) da equação 2 é uma codificação dos períodos anuais analisados. A análise foi realizada da seguinte forma:

- $1^{\circ}$ caso: Através da função inclinação, com os dados de preços reais de cada cultura e os valores de $t$ codificados (sendo 0 para o mês de janeiro 2002, 1 para o mês de fevereiro de 2002 e assim sucessivamente) determinou-se a tendência em termos monetários, para cada cultura. $\mathrm{O}$ valor obtido é o coeficiente angular da equação de regressão linear para os dados considerados. 
- $2^{\circ}$ caso: Utilizando a função inclinação, com os da dos de LN (Logaritmo Natural) dos preços das culturas analisadas e os valores de $t$ codificados (sendo 0 para o mês de janeiro 2002, 1 para o mês de fevereiro de 2002 e assim sucessivamente) determinou-se a tendência em termos percentuais, para cada cultura. Da mesma forma que descrito para o $1^{\circ}$ caso, o valor obtido é o coeficiente angular da equação de regressão linear para os dados considerados.

\section{RESULTADOS E DISCUSSÃO}

A partir dos históricos de preços obtidos para as principais culturas produzidas no Estado do Ceará comercializadas na CEASA - CE, Sede de Maracanaú, elaborou-se gráficos (Figuras 1 e 2) de evolução de preços ao longo do período de janeiro de 2002 à dezembro de 2014.

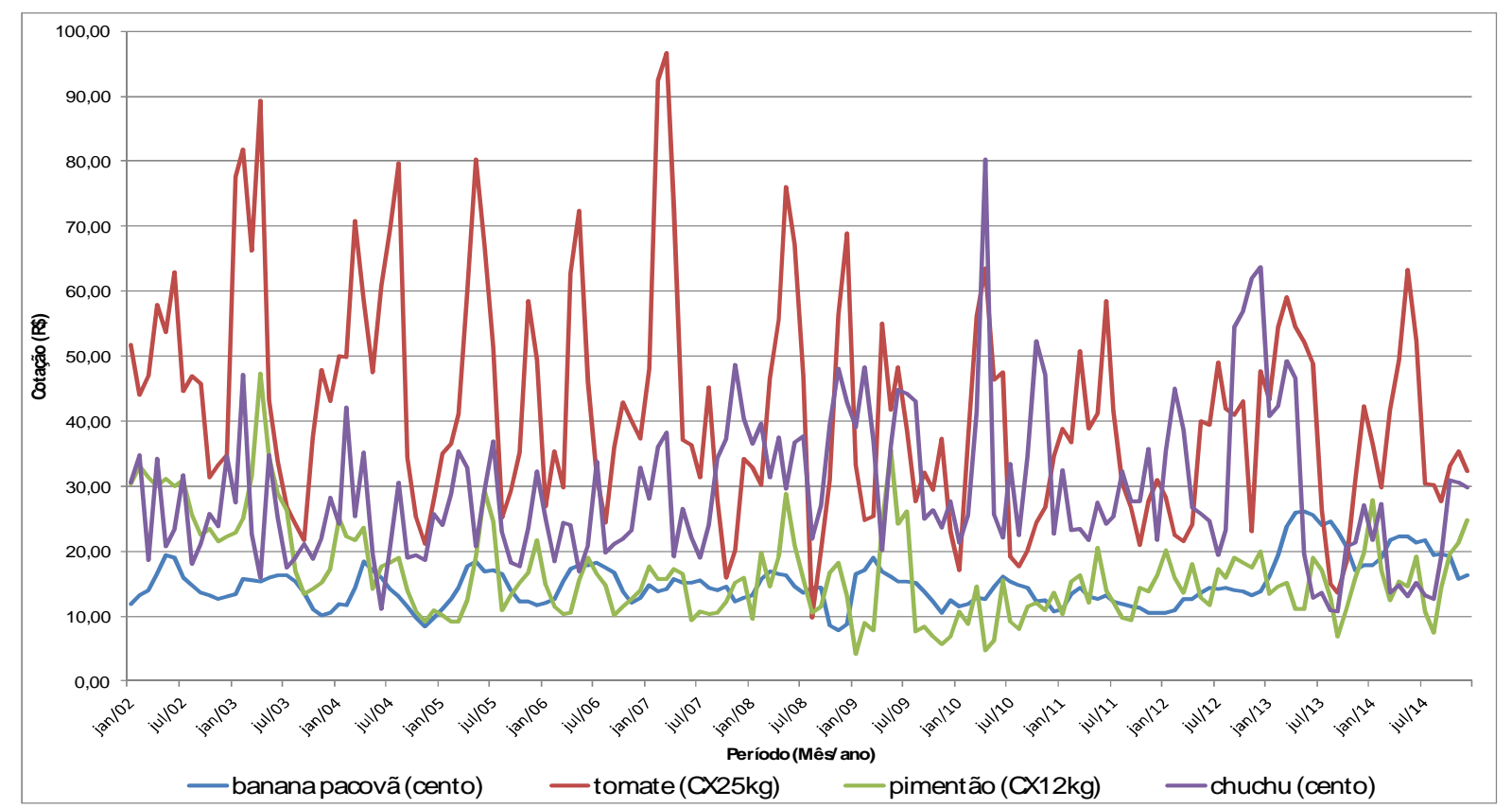

FIGURA 1 - Evolução dos preços de banana pacovan, tomate, pimentão e chuchu.

Fonte: Elaborado pelos Autores 


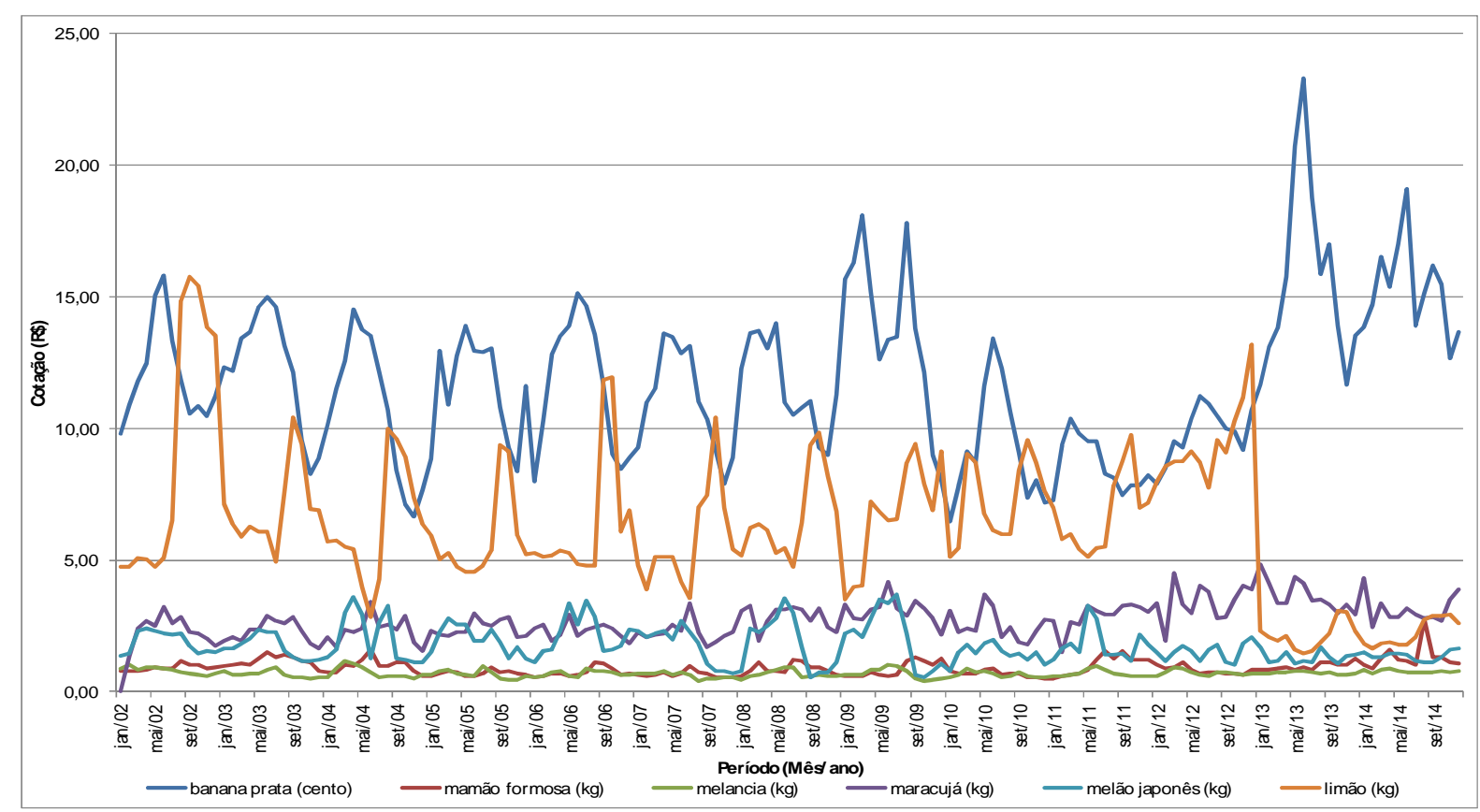

FIGURA 2 - Evolução dos preços das culturas de banana prata, mamão formosa, melancia, maracujá, melão japonês e limão.

Fonte: Elaborado pelos Autores

As análises das figuras 1 e 2 permitem observar que os preços exibem ciclos bem nítidos de elevação e queda e que nestes ciclos há uma significativa variabilidade entre os pontos de alta e baixa destes preços. Observe, por exemplo, as curvas definidas para as culturas de tomate e pimentão (figura 1), observa-se grande variação no preço do tomate entre os anos de 2006 e 2007, quando o preço atingiu o valor de $R \$ 24,42$ em agosto de 2006 e o valor $R \$ 96,70$ em março de 2007. Não obstante à variação verificada para a cultura do tomate, a cultura do pimentão também apresentou notável oscilação de preços ao longo do período analisado, entre os meses de janeiro e maio de 2009, por exemplo, o preço da caixa de $12 \mathrm{~kg}$ deste produto variou de $R \$ 4,09$ a $R \$ 35,58$. A Tabela 1 contém a estatística descritiva dos preços para cada cultura analisada.

TABELA 1 - Estatística descritiva por cultura da série mensal de preços analisada

\begin{tabular}{cccccc} 
Cultura & Média & Mínimo & Máximo & Desvio Padrão & $\begin{array}{c}\text { Coeficiente de } \\
\text { Variação (\%) }\end{array}$ \\
\hline Ban. pacovan (cento) & 14,98 & 7,86 & 26,08 & 9,18 & 18,77 \\
Tomate (cx25 kg) & 41,73 & 9,69 & 96,7 & 44,01 & 29,71 \\
Ban. Prata (cento) & 11,81 & 6,44 & 23,29 & 8,61 & 20,73 \\
Mamão formosa (kg) & 0,9 & 0,48 & 2,73 & 1,2 & 29,11 \\
Melancia (kg) & 0,69 & 0,4 & 1,16 & 0,38 & 16,85 \\
Pimentão (cx12 kg) & 16,52 & 4,09 & 47,33 & 22,26 & 32,77 \\
Maracujá (kg) & 2,74 & 1,36 & 4,83 & 1,75 & 19,56 \\
Chuchu (cento) & 28,95 & 10,76 & 80,2 & 36,01 & 30,03 \\
Melão japonês (kg) & 1,76 & 0,55 & 3,68 & 1,58 & 26,35 \\
Limão (kg) & 6,28 & 1,44 & 15,75 & 7,28 & 31,02 \\
\hline
\end{tabular}

Fonte: Elaborado pelos Autores 
Verifica-se, a partir da tabela 1, que as culturas de pimentão, chuchu e limão, seguido do tomate, possuem os maiores coeficientes de variação nos preços, com valores de: $32,77 \% ; 30,03 \% ; 31,02 \%$ e $29,71 \%$, respectivamente. Em contrapartida, a melancia apresentou o menor índice de coeficiente de variação entre todas as culturas analisadas, sendo igual a $16,85 \%$. Os maiores ou menores índices observados entre as culturas analisadas podem estar condicionados a fatores ambientais que exercem influência sobre o ciclo de determinadas culturas e também sobre decisões de mercado tomadas por produtores da cultura em questão, afetando diretamente ou indiretamente na variação dos preços no mercado.

Pode-se observar significativa variabilidade entre as culturas com destaque para o tomate, pimentão e chuchu, nestas culturas verificaram-se desvios padrão superiores a 20 , chegando a 44,01 na cultura do tomate. Obteve-se para as culturas de banana pacovan, banana prata e limão, desvios padrão menos acentuados, conforme segue: 9,$18 ; 8,61$ e 7,28 , respectivamente. Dentre os produtos analisados, a melancia apresentou o menor desvio padrão, 0,38.

Ainda analisando a tabela 1, pode-se observar que os produtos que apresentaram as maiores variações ou diferença entre o limite superior e inferior foram tomate e pimentão. As diferenças entre os limites superiores e inferiores atingiram a média de $212,57 \%$ para o tomate e $212,66 \%$ para o pimentão. Em contrapartida, as menores diferenças foram obtidas nas culturas de banana pacovan e banana prata, $37,64 \%$ e $57,27 \%$, respectivamente, e na cultura da melancia com $61,38 \%$. Quanto maior essa diferença percentual maior a variação nos preços, sendo o inverso verdadeiro.

Vale ressaltar que as menores diferenças entre os limites superiores e inferiores não correspondem exatamente aos menores índices de coeficiente de variação. Conforme mencionado anteriormente, as menores diferenças foram obtidas para as culturas da banana pacovan, banana prata e melancia, observandose, nestas culturas, diferenças de: $37,64 \% ; 57,27 \%$ e $61,38 \%$, respectivamente. Os índices de coeficiente de variação obtidos para essas culturas foram $18,77 \%$ para a banana pacovan, $20,73 \%$ para a banana prata e $16,85 \%$ para a melancia.

É importante frisar que os valores dos coeficientes de variação obtidos foram determinados considerando a média geral, o valor mínimo e o valor máximo de toda série de preço analisada.

\section{Análise de sazonalidade de frutas}

A tabela 2 contém os índices sazonais das culturas de banana pacovan, banana prata, mamão formosa, melancia, maracujá, melão japonês e limão. Além dos índices sazonais, podem-se observar também os limites inferiores e superiores mensais de IEP, considerando cada uma das culturas analisadas neste tópico. 
TABELA 2 - Índices sazonais, limites inferiores e superiores das frutas analisadas, relativos à variação de preços entre 2002 - 2014, CEASA - CE, 2015

\begin{tabular}{|c|c|c|c|c|c|c|c|c|c|c|c|c|}
\hline \multirow{2}{*}{ Mês } & \multicolumn{3}{|c|}{ Banana Pacovan } & \multicolumn{3}{|c|}{ Banana Prata } & \multicolumn{3}{|c|}{ Mamão formosa } & \multicolumn{3}{|c|}{ Melancia } \\
\hline & $\mathrm{I}_{\text {sazonal }}$ & L. Sup. & L. Inf. & $\mathrm{I}_{\text {sazonal }}$ & L. Sup. & L. Inf. & $\mathrm{I}_{\text {sazonal }}$ & L. Sup. & L. Inf. & $I_{\text {sazonal }}$ & \multirow{2}{*}{$\begin{array}{c}\text { L. Sup. } \\
108,77\end{array}$} & L. Inf. \\
\hline Jan & 89,50 & 116,71 & 80,45 & 86,95 & 122,34 & 58,27 & 82,90 & 103,96 & 65,75 & 90,00 & & 72,27 \\
\hline $\mathrm{Fev}$ & 97,20 & 119,23 & 84,62 & $100,40 *$ & 121,74 & 71,81 & 82,83 & 100,75 & 67,08 & 99,99 & 122,19 & 87,07 \\
\hline Mar & $108,74^{*}$ & 134,09 & 95,73 & $108,65^{*}$ & 133,02 & 90,00 & 95,03 & 136,63 & 76,17 & $114,42 *$ & 163,30 & 88,94 \\
\hline Abr & $114,90^{*}$ & 141,03 & 96,21 & $111,79 *$ & 133,95 & 89,95 & 98,53 & 124,11 & 75,08 & $117,15^{*}$ & 153,03 & 101,99 \\
\hline Mai & $116,46^{*}$ & 132,35 & 104,39 & $119,48^{*}$ & 137,77 & 92,33 & 95,81 & 120,80 & 78,94 & $111,96 *$ & 130,78 & 91,01 \\
\hline Jun & $114,35^{*}$ & 124,08 & 105,02 & $121,54 *$ & 151,59 & 96,20 & $104,17 *$ & 164,28 & 69,09 & $112,96 *$ & 152,70 & 81,15 \\
\hline Jul & $110,36^{*}$ & 119,06 & 99,31 & $113,05^{*}$ & 134,24 & 87,98 & $109,22 *$ & 148,14 & 76,83 & $115,17 *$ & 147,15 & 77,06 \\
\hline Ago & $106,15^{*}$ & 114,42 & 98,28 & $105,75^{*}$ & 141,13 & 88,34 & $122,41 *$ & 149,25 & 93,81 & 95,85 & 114,62 & 69,13 \\
\hline Set & 98,09 & 107,20 & 88,12 & 93,95 & 114,55 & 78,28 & $116,60^{*}$ & 150,35 & 86,47 & 89,97 & 115,15 & 70,39 \\
\hline Out & 85,67 & 98,21 & 61,12 & 82,20 & 107,04 & 66,17 & $105,83 *$ & 135,09 & 74,79 & 84,35 & 104,18 & 59,68 \\
\hline Nov & 77,76 & 92,47 & 55,54 & 74,57 & 90,84 & 62,14 & 93,90 & 114,79 & 75,74 & 80,58 & 91,81 & 63,96 \\
\hline \multirow[t]{15}{*}{ Dez } & 80,82 & 92,89 & 62,32 & 81,68 & 99,05 & 72,00 & 92,77 & 141,92 & 67,69 & 87,59 & 98,84 & 69,73 \\
\hline & \multirow{2}{*}{\multicolumn{2}{|c|}{ Mês }} & \multicolumn{3}{|c|}{ Maracujá } & \multicolumn{3}{|c|}{ Melão Japonês } & \multicolumn{3}{|c|}{ Limão } & \\
\hline & & & $\mathrm{I}_{\text {sazonal }}$ & L. Sup. & L. Inf. & $\mathrm{I}_{\text {sazonal }}$ & L. Sup. & L. Inf. & $\mathrm{I}_{\text {sazonal }}$ & L. Sup. & L. Inf. & \\
\hline & & Jan & 88,51 & 126,22 & 59,26 & 82,26 & 120,73 & 41,94 & 81,10 & 105,11 & 40,64 & \\
\hline & & $\mathrm{Fev}$ & 95,66 & 137,58 & 72,70 & 96,25 & 128,96 & 72,94 & 76,37 & 101,53 & 39,50 & \\
\hline & & Mar & 96,44 & 102,73 & 89,75 & $112,41 *$ & 149,86 & 77,92 & 81,81 & 123,95 & 41,34 & \\
\hline & & Abr & $110,96^{*}$ & 147,48 & 89,67 & $124,37 *$ & 183,77 & 90,50 & 84,79 & 118,85 & 50,51 & \\
\hline & & Mai & $118,78^{*}$ & 149,59 & 92,27 & $135,74 *$ & 191,29 & 76,96 & 79,96 & 104,46 & 43,81 & \\
\hline & & Jun & $105,71^{*}$ & 143,83 & 82,37 & $124,45^{*}$ & 201,19 & 63,18 & 76,16 & 96,81 & 44,87 & \\
\hline & & Jul & $103,59 *$ & 123,61 & 78,02 & $124,18^{*}$ & 183,90 & 78,28 & 77,35 & 99,56 & 61,89 & \\
\hline & & Ago & 99,14 & 125,01 & 70,97 & $111,15^{*}$ & 158,70 & 79,29 & $112,93 *$ & 163,63 & 77,11 & \\
\hline & & Set & $100,03 *$ & 122,48 & 72,59 & 72,18 & 100,70 & 27,95 & $141,90 *$ & 190,89 & 108,96 & \\
\hline & & Out & 90,40 & 109,23 & 79,48 & 61,06 & 80,20 & 29,79 & $149,77^{*}$ & 192,58 & 105,07 & \\
\hline & & Nov & 84,16 & 110,86 & 64,59 & 77,32 & 127,62 & 35,84 & $120,63 *$ & 163,75 & 91,06 & \\
\hline & & Dez & $106,63 *$ & 135,27 & 83,09 & 78,61 & 149,01 & 35,74 & $117,23 *$ & 210,89 & 88,29 & \\
\hline
\end{tabular}

Fonte: Elaborado pelos autores

Verifica-se que todas as culturas possuem, nos meses finais do ano, baixas de preços, sendo que a banana prata apresentou o período mais longo de alta nos preços, indo de fevereiro até agosto.

A partir da análise da tabela 2 pode-se verificar que no início do ano (especificamente os meses de janeiro e fevereiro) as culturas de banana pacovan, mamão formosa, melancia, maracujá, melão japonês e limão, mantiveram-se abaixo da média, isto é, possuem preços mais baixos no início do ano, enquanto a cultura de banana prata exibe no mês de janeiro índices inferiores à média, superando esta no mês de fevereiro. DJAU et al. (2014) encontraram resultados semelhantes para a cultura da banana prata quando avaliaram a variação de preços de frutas no Estado do Ceará. 


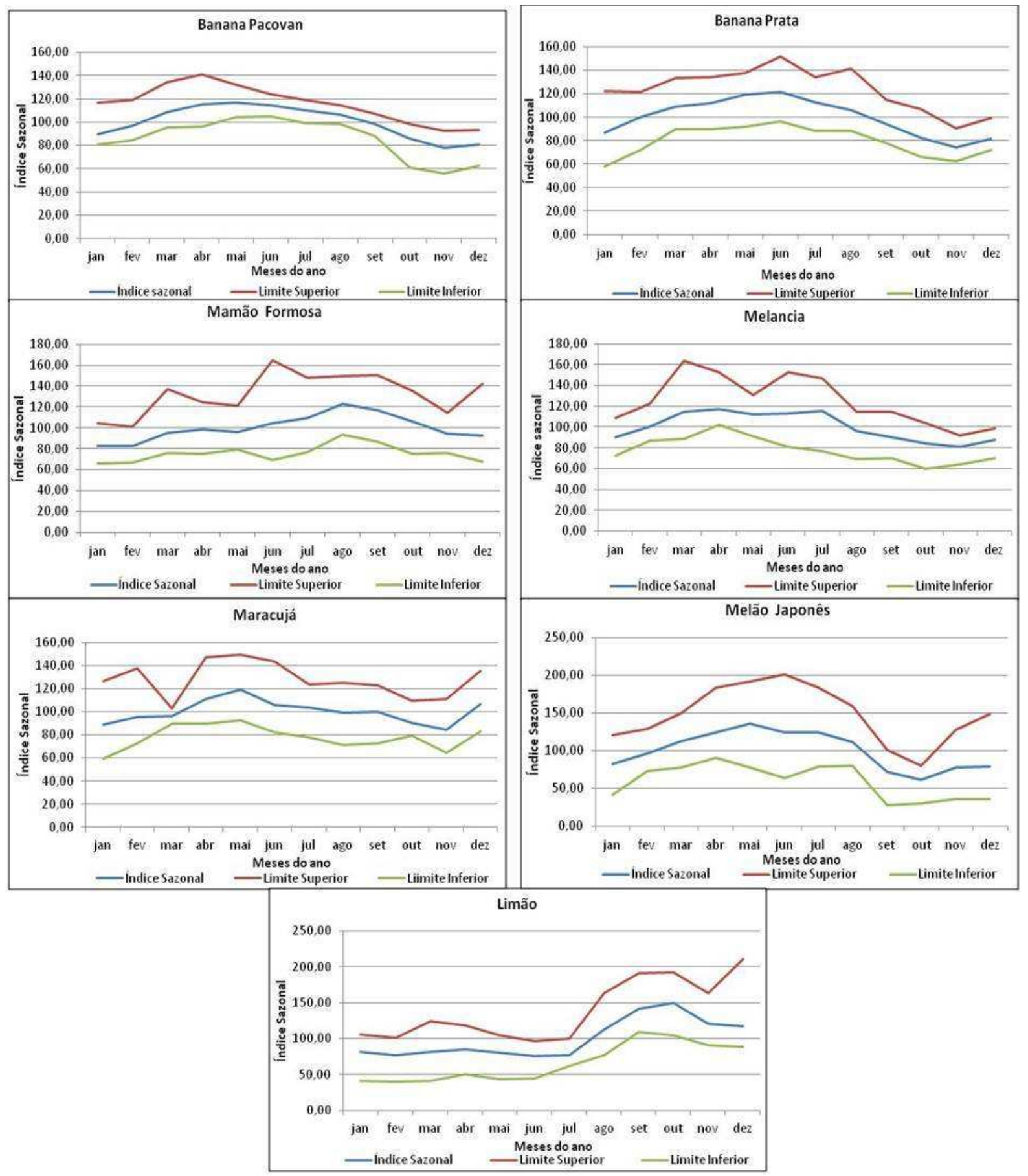

FIGURA 3 - Índices sazonais, limites inferiores e superiores da banana pacovan, banana prata, mamão formosa, melancia, maracujá, melão japonês e limão, relativos à variação de preços calculada para os 13 anos, 2002 2014, CEASA - CE, 2015.

Fonte: Elaborado pelos Autores

Analisando a figura 3 pode-se observar que os limites inferiores e superiores oscilam aproximadamente na mesma proporção que as linhas dos índices sazonais dos preços analisados, salvo pontos em que há picos de alta ou baixa. Para a banana pacovan, observa-se que esta inicia o ano em baixa de preços, porém no 
mês de março os preços já atingem níveis acima da média e se mantém desta forma até o mês de agosto, quando declina novamente. Os picos observados nos gráficos da figura 3 podem ser ocasionados por fatores ambientais e também por decisões de planejamento por parte dos produtores que podem interferir nos preços destas culturas, como o foco no mercado internacional, por exemplo, no caso da banana.

A banana é produzida o ano inteiro, mas dependendo da variedade e da região pode exibir picos de oferta em determinados meses, como ocorre com as variedades pacovan e prata, ambas analisadas neste trabalho. Esta última, assim como a banana pacovan, apresentou padrão de sazonalidade nos preços praticados na comercialização na CEASA - CE. A banana prata inicia o ano com o nível de preços abaixo da média (mês de janeiro), porém logo no mês de fevereiro os preços se elevam e mantêm-se acima da média até o mês de agosto, estes então caem e fecham o ano em baixa.

A maioria das culturas demonstraram períodos de alta e baixa de preços bem definidos, com exceção do maracujá, que possui período de entressafra nítido entre os meses de abril a julho, porém no mês de setembro o preço deste produto também está em alta, estando este mês entre dois períodos de baixa de preços, fato que se repete no mês de dezembro.

O mamão formosa possui período de entressafra que vai de junho a outubro. Durante boa parte do primeiro semestre (janeiro a maio) o mamão apresenta-se com preços abaixo da média, havendo uma subida nos preços no mês de junho, como citado anteriormente, e voltando a cair novamente a partir do mês de novembro.

Já para a melancia verificou-se um comportamento semelhante à cultura do tomate, na qual em boa parte do primeiro semestre os preços mantêm-se acima da média, caracterizando um cenário de entressafra. Este fato, a exemplo do que ocorre com o tomate, pode estar relacionado intimamente à quadra chuvosa vigente no Estado que, caracteristicamente, ocorre no primeiro semestre do ano. Especificamente, o período de alta nos preços ocorre no período de março a julho.

O melão japonês evidencia um período de entressafra entre os meses de março e agosto, que é o período de maiores níveis de preços. Este resultado se aproxima dos resultados encontrados por pesquisadores do CEPEA (2015) em um estudo realizado para definir as melhores épocas de comercialização para o melão japonês no mercado do Estado de São Paulo. Naquele trabalho eles identificaram como período de melhores preços, o período de fevereiro a julho, por este ser o período de entressafra no principal pólo produtivo nacional que é pólo RN/CE. Neste período costuma ser chuvoso nessa região, o que impede a colheita em larga escala deste produto.

A cultura do limão exibe um comportamento sazonal bem característico, onde durante todo o primeiro semestre, os níveis de preços estão abaixo da média, especificamente do início do ano até o mês de julho, isto porque este período corresponde à safra da cultura. Em contrapartida, a partir do mês de agosto até o final do ano, este produto possui os maiores índices de preços, correspondendo, portanto, ao período de entressafra da cultura.

\section{Análise de sazonalidade de hortaliças-fruto}

$\mathrm{Na}$ tabela 3 estão contidos os índices sazonais, limites inferiores e limites superiores mensais de IEP para as culturas do tomate, pimentão e chuchu. 
TABELA 3 - Índice sazonal, limites inferiores e superiores do tomate, pimentão e chuchu, no período de 2002 - 2014, CEASA - CE, 2015

\begin{tabular}{cccccccccc}
\hline \multirow{2}{*}{ Mês } & \multicolumn{3}{c}{ Tomate } & \multicolumn{3}{c}{ Pimentão } & \multicolumn{3}{c}{ Chuchu } \\
\cline { 2 - 10 } & $I_{\text {sazonal }}$ & L. Sup. & L. Inf & $\mathrm{I}_{\text {sazonal }}$ & L. Sup. & L. Inf & $\mathrm{I}_{\text {sazonal }}$ & L. Sup. & L. Inf. \\
\cline { 2 - 10 } Jan & 92,69 & 153,97 & 46,74 & 98,48 & 179,78 & 24,6 & $103,51^{*}$ & 126,51 & 63,2 \\
Fev & $104,87^{*}$ & 187,51 & 66,91 & 99,54 & 126,62 & 55,94 & $125,94^{*}$ & 183,89 & 77,35 \\
Mar & $122,27^{*}$ & 195,11 & 65,46 & $100,14^{*}$ & 161,21 & 49,28 & $109,22^{*}$ & 143,4 & 76,34 \\
Abr & $139,82^{*}$ & 193,1 & 72,78 & $109,36^{*}$ & 187,61 & 50,4 & $105,98^{*}$ & 238,79 & 52,99 \\
Mai & $131,75^{*}$ & 187,67 & 80,34 & $121,75^{*}$ & 243,13 & 64,47 & 83,82 & 137,2 & 61,64 \\
Jun & $123,64^{*}$ & 159,22 & 69,96 & $123,54^{*}$ & 187,99 & 67,77 & 81,39 & 127,08 & 44,22 \\
Jul & 95,19 & 143,03 & 54,31 & $113,89^{*}$ & 191,69 & 78,44 & 95,01 & 141,42 & 48,84 \\
Ago & 75,95 & 167,99 & 22,22 & 82,05 & 122,58 & 54,59 & 81,19 & 132,35 & 43,98 \\
Set & 68,01 & 100,73 & 37,82 & 77,76 & 119,44 & 45,27 & 85,71 & 134,15 & 47,37 \\
Out & 68,2 & 99,94 & 42,42 & 86,2 & 115,42 & 48,87 & 99,63 & 154,21 & 70,23 \\
Nov & 83,16 & 131,74 & 44,29 & 88,32 & 114,91 & 44,55 & $112,16^{*}$ & 158,02 & 66,27 \\
Dez & 94,46 & 169,4 & 56,84 & 98,98 & 131,73 & 61,53 & $116,45^{*}$ & 160,63 & 71,85 \\
\hline
\end{tabular}

Isazonal: indice Sazonal

L. Sup : : Limite superior

L. Inf: Limite Inferior

Na coluna dos índices sazonais, os valores seguidos de asteriscos $\left(^{*}\right)$ correspondem à períodos de entressafra, isto é, indice sazonal maior que 100.

Fonte: Elaborado pelos Autores

Observando a tabela 3, verifica-se que a cultura do pimentão inicia o ano em baixa de preços. O mesmo ocorre para a cultura do tomate, que demonstra índice sazonal menor que 100 no mês de janeiro, mas superando este logo no mês de fevereiro. O chuchu apresenta um comportamento diferente, uma vez que inicia o ano em alta, permanecendo assim até o mês de abril, quando ocorre uma queda nos preços, voltando a subir novamente a partir de novembro. Vale ressaltar que a cultura do chuchu fecha o ano em alta de preços, o que não ocorre para as culturas de tomate e pimentão.

$\mathrm{Na}$ figura 4, de maneira análoga à descrição proposta para a figura 3, estão expostas as linhas dos índices sazonais, dos limites superiores e inferiores. Observa-se que, salvo oscilações de picos, as linhas dos limites superiores e inferiores variam aproximadamente na mesma proporção que as linhas dos índices de sazonalidade.

O tomate inicia o ano em baixa, com níveis de preços abaixo da média, porém logo em fevereiro, a exemplo da banana prata, atinge níveis de preços mais elevados, permanecendo em alta até o mês de junho, quando então declina novamente. Um fato que pode estar relacionado à alta dos preços na cultura do tomate nos meses citados é a coincidência destes com a quadra de chuvas que ocorre no Estado do Ceará, normalmente nos primeiro semestre do ano. O período de molhamento foliar, ocasionado pelas chuvas a que as plantas geralmente são submetidas quando cultivadas no campo (situação da maior parcela dos cultivos desta cultura no Ceará), favorece a infecção e disseminação de doenças, podendo causar elevadas perdas na produção e sem a adoção de um manejo de controle adequado, as pragas também podem causar danos consideráveis à cultura e queda na produção conforme cita GALLO et al. (2002). Estas perdas e o aumento nos 
custos de produção diminuem a oferta deste produto no mercado, aumentando o nível de preços, conforme pode-se observar na tabela 4.

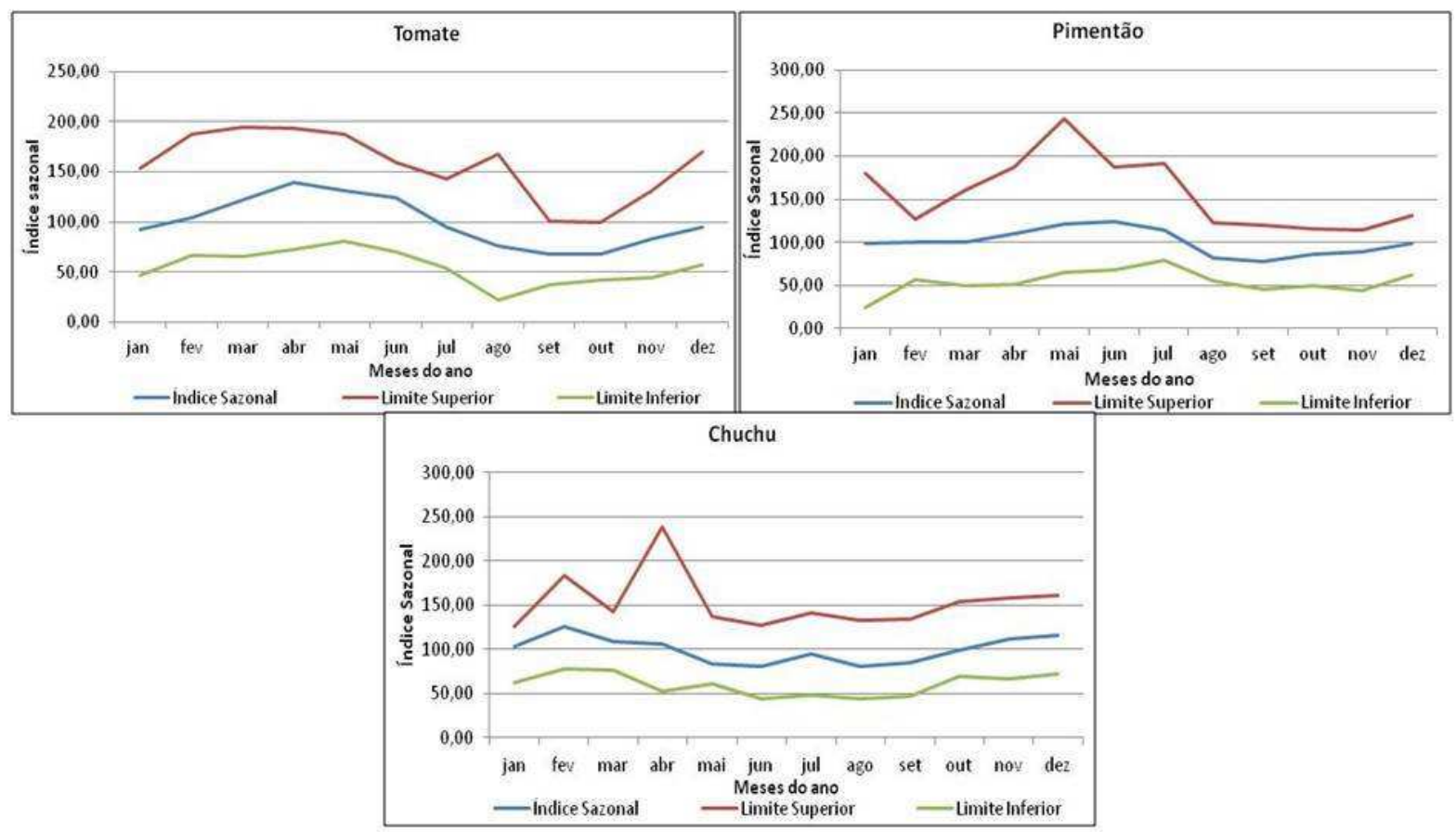

FIGURA 4 - Índices sazonais, limites inferiores e superiores do tomate, pimentão e chuchu, relativos à variação calculada para os 13 anos, 2002 - 2014. CEASA - CE, 2015.

Fonte: Elaborado pelos Autores.

A cultura do pimentão possui um comportamento semelhante ao descrito para a cultura do tomate e melancia, certamente pelos mesmos motivos. Isto é refletido diretamente nos preços praticados na comercialização deste produto, uma vez que a diminuição da oferta do produto no mercado ocasiona uma elevação nos níveis de preços.

O chuchu inicia o ano em alta, com níveis de preços que permanecem acima da média até o mês de Abril. A partir daí os preços caem, permanecendo em baixa até o mês de outubro, quando se elevam novamente e fecham o ano em alta.

\section{Análise de Tendência}

A análise da Tabela 4 permite observar que algumas culturas exibiram tendência de preços negativa e que o restante das culturas analisadas apresentam tendências positivas de crescimento (considerando os dados de preços mensais).

Os produtos para os quais se obtiveram tendência de queda nos preços foram: tomate, melancia (em termos monetários), pimentão, melão japonês e limão. A desvalorização destes produtos, considerando o período utilizado para a análise, está correlacionada diretamente com o aumento da produtividade e da oferta dos produtos citados. 
TABELA 4 - Tendência em termos monetários e percentuais por cultura

\begin{tabular}{ccc}
\hline Cultura & Tendência (t. Monetários) & Tendência (t. Percentuais) \\
\hline Banana pacovan & 0,026 & 0,144 \\
Tomate & $-0,108$ & $-0,252$ \\
Banana prata & 0,009 & 0,051 \\
Mamão formosa & 0,001 & 0,072 \\
Melancia & $-3,97 \mathrm{e}^{-05}$ & 0,009 \\
Pimentão & $-0,061$ & $-0,32$ \\
Maracujá & 0,009 & 0,307 \\
Chuchu & 0,026 & 0,027 \\
Melão japonês & $-0,004$ & $-0,248$ \\
Limão & $-0,02$ & $-0,472$ \\
\hline
\end{tabular}

Fonte: Elaborado pelos Autores.

A adoção de novas tecnologias de produção, possibilitando maior produtividade por área e maior interesse empresarial pela produção e distribuição de hortifrutigranjeiros, são elementos importantes para entender a queda significativa de preços. O aumento na oferta de produtos e o incremento de renda obtidos a partir do Plano Real estimularam o maior consumo por partes dos consumidores (VIANA et al., 2010). Novas formas de processamento de produtos hortigranjeiros permitem a comercialização em escala destes produtos, o que em uma escala global, resulta em uma redução do preço unitário do produto. CARDOSO et al. (2000) já destacavam a tendência de aumento do consumo de bebidas não alcoólicas, que de certa forma usam na sua preparação uma quantidade relevante de frutas de clima tropical, o que indiretamente influencia na formação dos preços destes produtos.

Analisando a cultura do maracujá, por exemplo, verifica-se que esta cultura possui uma tendência em termos monetários de 0,009 indicando que o preço do $\mathrm{kg}$ deste produto cresce, mensalmente, $\mathrm{R} \$ 0,009$. Em termos percentuais 0 crescimento foi de $0,307 \%$ ao mês. Este raciocínio deve ser aplicado às demais culturas analisadas. A cultura da melancia possui tendência de preço praticamente igual à zero, isto é, tende a ter alterações mínimas em seus preços de comercialização.

O restante das culturas apresentou tendência de crescimento dos preços, como a banana pacovan e o maracujá, por exemplo. A maior demanda por estes produtos no mercado interno, aliado ao crescente interesse dos produtores de atingir o mercado internacional, pode justificar essa tendência de aumento de preços nessas culturas. Além da banana pacovan, que tem uma tendência, em termos monetários, de 0,026 (mesma tendência de preços verificada para a cultura do chuchu sendo o maior valor entre as culturas analisadas) e do maracujá, as culturas de banana prata e mamão formosa, também demonstraram tendência de crescimento de preços, conforme apresentou a tabela 4. 


\section{CONCLUSÕES}

Todas as culturas analisadas apresentaram padrão de sazonalidade em seus preços de comercialização no mercado da CEASA - CE, Sede Maracanaú.

Cinco entre os dez produtos analisados exibiram tendência de crescimento negativa, são eles: tomate, melancia, pimentão, melão japonês e limão. $\mathrm{O}$ restante dos produtos: banana pacovan, maracujá, banana prata, mamão formosa e chuchu, demonstraram tendência positiva de crescimento dos preços.

Os resultados obtidos podem permitir um melhor planejamento da produção por parte de produtores e uma melhor programação financeira de consumidores, de uma forma geral.

\section{REFERÊNCIAS}

BARROSO NETO, A. M.; ARAÚJO, L. B. R.; SOUSA, A. M.; VASCONCELOS, M. R. A.; BLEICHER, E. Fenologia de produção e comportamento de preços da berinjela no estado do ceará. Enciclopédia Biosfera, Centro Científico Conhecer - Goiânia, $\begin{array}{lllll}\text { v.11 n.22; } & \text { p. } & 1264 . & 2015 . & \text { DOI: }\end{array}$ http://dx.doi.org/10.18677/Enciclopedia_Biosfera_2015_157.

BENTO, D. G. C.; TELES, F. L. A sazonalidade da produção agrícola e seus impactos na comercialização de insumos. Revista Científica do Centro de Ensino Superior Almeida Rodrigues. Rio Verde, FAR/ISEAR, Ano 1, no 1, 2013.

CARDOSO, C. E. L.; SOUSA, J. S. Fruticultura tropical: perspectiva e tendências. Revista Econômica do Nordeste, v.31, n.1, p.84-95. 2000. Disponível em: http://www.bnb.gov.br/projwebren/Exec/artigoRenPDF.aspx?cd_artigo_ren=193. Acessado em: 11 dez. 2015.

CARDOSO, M. V.; SOARES, P. R. C.; SILVA, J. C. G. L.; TIMOFEICZYK JUNIOR, R. Estudo da Sazonalidade de preço da celulose brasileira no mercado dos Estados Unidos em períodos cíclicos como apoio a estratégias empresariais. Scientia Forestalis, v. 41. n. 97. p. 47-55. 2013. Disponível em: http://www.ipef.br/publicacoes/scientia/nr97/cap05.pdf. Acessado em: 12 dez. 2015.

CENTRAIS DE ABASTECIMENTO DO CEARÁ - CEASA. 2013. Disponível em: http://www.ceasa-ce.com.br/index.php/historicoofertas. Acesso em: 28 nov. 2015.

CENTRO DE ESTUDOS AVANÇADOS EM ECONOMIA APLICADA - CEPEA ESALQ/USP. 2005. Qual a melhor época a venda de hortifrutícolas? $n^{\circ} 142$, ISSN 1981- $1837 . \quad$ Disponível em: http://www.cepea.esalq.usp.br/hfbrasil/edicoes/142/full.pdf. Acessado em: $10 \mathrm{dez}$. 2015.

FACHINELLO, J. C.; PASA, M. S.; SCHMTIZ, J. D.; BETEMPS, D. L. Situação e perspectivas da fruticultura de clima temperado no Brasil. Revista Brasileira de Fruticultura, v.33, p. 109-120. 2011. Disponível em: http://www.scielo.br/pdf/rbf/v33nspe1/a14v33nspe1.pdf. Acessado em: 12 dez. 2015.

GALLO, D. et al. Entomologia Agrícola. Piracicaba: FEALQ, 920 p. 2002.

HARZER, J. H.; COSTA, C. T.; SILVA, W.V.; SOUZA, A. Eficiência dos mercados futuros de commodities agrícolas aplicando-se o teste de cointegração. Revista 
de Administração da Universidade Federal de Santa Maria. Santa Maria. v. 5. n. 2.p. 336-353. 2012.

DJAU, M. A.; REIS, J. N. P.; LIMA, P. V. P. S. Análise de variação de preços de frutas no estado do ceará de 2007 a 2011. Enciclopédia Biosfera, Centro Científico Conhecer - Goiânia, v.10, n.18, p. 3434. 2014. Disponível em: http://www.conhecer.org.br/enciclop/2014a/CIENCIAS\%20EXATAS/analise.pdf.

Acessado em: 12 dez. 2015.

MARQUES, P. V.; AGUIAR, D. R. D. Comercialização de Produtos Agrícolas. Edusp, São Paulo. 295 p. 1993.

MARQUES, R. W. C.; CAIXETA FILHO, J. V. Sensonlity of the flowers and ornamental market in São Paulo state: the case of CEAGESP-SP. Revista de Economia e Sociologia Rural. V.40, n. 4, p. 789-806, ISSN 1806-9479. 2002. Disponível em:

http://dx.doi.org/10.1590/S0103-20032002000400003. Acessado em: 18 dez. 2015.

MENDES, J. T. G.; PADILHA JUNIOR, J. B. Agronegócio: uma abordagem econômica. São Paulo: Pearson Prentice Hall, 1 ed. 384 p. 2007.

SOUSA, A. M.; BARROSO NETO, A. M.; PINHEIRO, J. I.; SILVA, V. B.; NOGUEIRA, D. B. Comportamento dos preços de milho e feijão caupi no mercado do estado do Ceará. Revista Verde, vol. 10, № 5 (ESPECIAL), p. 01 - 08. 2015. DOI: http://dx.doi.org/10.18378/rvads.v10i5.3399.

SPIEGEL, M. R. Estatística. 3 ed. São Paulo: Makron Books. 643 p. 1993.

VIANA, J.G.A.; BARCHET, I.; ZEN, B.; SOUZA, R. S. Tendência histórica de preços pagos ao produtor de hortifrutigranjeiros do Rio Grande do Sul, Brasil. Ciência Rural. Santa Maria. v.40. n.7. p. 1643-1650. 2010. 